\title{
Fundamental Geodetic Parameters 1999
}

\author{
Erwin Groten \\ Univ. Technology, Darmstadt, Germany
}

\begin{abstract}
The present (2000) set of primary "constants" and "current best estimates" is being reviewed. The emphasis within IUGG is on the consideration and numerical evaluation of temporal changes of relevant parameters. The accuracy of many parameters has been substantially improved, but many numerical values did not change significantly. Therefore, to avoid confusion IUGG/IAG are reluctant in introducing new official parameter sets but keep "current best estimates" up-to-date.
\end{abstract}

\section{Introduction}

The report on the work of the Special Commission 3 "Fundamental Parameters" of the International Association of Geodesy (IAG) as given at IAU Colloquium 180 in Washington in spring 2000 represents the present situation within IUGG and IAG. It appeared appropriate to wait for new systems to be adopted within IAU including new nutation formulas, precession constant etc. before new geodetic parameter sets can be derived.

In general, the tendency in IAG is not to change too often the sets of primary constants but to keep sets of "current best estimates" up-to-date and available to the scientific community. A typical example is the ellipsoidal "Geodetic Reference System 1980" where, meanwhile, the main ellipsoidal parameters are "off" by more than $0.4 \mathrm{~m}$. In view of temporal change of the Earth's spin vector $\vec{\omega}$ within $\pm 10^{-8}$ only a truncated value is being used as a defining constant. Consequently, all 4 fundamental parameters adopted in resolution 2 of IUGG, are meanwhile more or less "conventional" parameters. In spite of the consequent deficiencies it appeared still appropriate not to introduce a new official ellipsoidal (level ellipsoid) system even though new systems of that kind have been derived in terms of GRD 2000 etc. and, consequently, are available to those who may need such systems for various practical reasons. Whether or not IUGG will decide after the Manchester General Assembly of IAU to consider the new parameters and basically new systems to be adopted here and, together with IERS, will propose a new set of geodetic systems and parameters to the next IUGG General Assembly to be held in 2003 in Japan, is a different matter. In combination with IAU, to revise all relevant parameters would make sense, where, for IUGG, the temporal changes which can now be derived due to substantially increased accuracy would be a vital aspect so that many "constants" had to be associated with corresponding temporal gradients.

A typical example, where IUGG had to wait for a new IAU-parameter set is the dynamic ellipticity $H$ of the Earth which, as already pointed out at IAU 
Colloquium 180, is depending on the precession constant. With better and new Earth models available this quantity, which depends basically on the difference between polar and equatorial moments of inertia, can be substantially improved.

In principle, $H$ can be directly derived as an output of the new precession nutation formula system as evaluated by Prof. P. Mathews. On the other hand, there is no longer a unique Earth model associated with that formula. This is different from the previous nutation formula by $\mathrm{J}$. Wahr and the precession constant was in the past derived more or less independently as an empirical quantity. This and similar questions raise new problems which need to be carefully investigated. Thus a permanent discussion between IAU, IUGG, including IERS, appears necessary.

One of the most problematic "constants" is Newton's gravitational constant $G$ where $G M$ (with $M=$ mass of the Earth including the atmosphere) has been derived with very high accuracy by satellite techniques whereas recent determinations of $G$ itself tend to reveal quite diverging results.

For geodetic purposes the new model of precession-nutation by P.M. Mathews, T.A. Herring and B.A. Buffett is of prime interest, as far as new reference systems to be adopted by IAU are concerned: A typical example is the new dynamic ellipticity (Mathews et al. 2000),

$$
H=(C-A) / C=3.2737875 \times 10^{-3}
$$

which significantly deviates from the "best estimates" such as

$$
\begin{aligned}
& H=3.273763(0.000020) 10^{-3} \text { by IAG, and } \\
& H=3.273668, H=3.273548, H=3.273674
\end{aligned}
$$

by authors such as Bretagnon, Dehant etc. The fact that in the semi-empirical approach by MHB there is no underlying unique Earth-model to which the MHB value of $H$ can be referred, makes comparisons with previous values of $H$ difficult. As is seen above the MHB differs by more than the standard deviation (one sigma) from the IAG-value, which is related to the well-known "corrected Fricke" value of the precession constant. It is now difficult to say, in view of the semi-empirical results, whether the precession or a different Earth model causes the difference. This deserves further investigation.

If, in the new definition of TT, the atomic time is decoupled from the geopotential $W^{o}$ at the geoid we should recognize that still potential differences can be identified with time differences but the relation to the geoid is lost so that geoid heights can no longer be determined from transportable clock readings. However, geoid height differences become available from time readings. This means that absolute readings or measurements are replaced by relative data.

In order to better understand the fundamental difference between absolute and relative geoid determination the exact geoid definition and its implementation is given in an annex.

However, the desired accuracy of the time measurement approach is only achieved within the frame of general relativity when accuracy in relative time observations of better than $10^{-15}$ will be achieved. In so far the new attempts in IAU to create a fully relativistic set of reference frames is of utmost importance for global geodesy where presently accuracies of the order of up to $10^{-9}$ are standard. Within consequent positioning in such frames with centimeter accuracy even now special and general relativistic reductions are common practice. 
A typical example is timing using GPS time for DGPS and similar requirements for timing in VLBI-applications.

In order to illustrate the present situation the most recent catalogue of "current best estimates" and related accuracies and variations with time for fundamental geodetic parameters is given in (Groten, 2000).

In this connection the basic difference between "current best estimates" and sets of fundamental parameters, also in view of their temporal variations, must be pointed out. This fact is all the more important as fundamental parameters, such as $G M, a, W^{o}$, are often derived now from one and the same set of satellite data, so that $G M$ (geocentric gravitational constant) and $a$ (semi-major axis of the Earth) are no longer independent. A similar problem arises with the aforementioned values of $H$. These inconsistencies are often unavoidable in sets of "current best estimates" but should be abolished in fundamental data sets.

\section{Annex: Definition of the Geoid}

Let $W(r, \theta, \lambda)=$ geopotential with geocentric spherical coordinates $(r, \theta, \lambda)$; $W^{o}=$ geopotential at the geoid $(S)$;

$U=$ normal potential defined by $\left(G M, \omega, J_{2}, a\right)$,

with $a=$ semi-major axis of a level ellipsoid $(E), J_{2}=$ second zonal harmonic of $W, \omega=$ spin rate of Earth's rotation, $G M=$ geocentric gravitational constant.

$$
\begin{gathered}
T=W-U \\
W^{\circ}-U^{o}=0 \text { with } W^{\circ}=W(S) \text { and } U^{o}=U(E) \\
\lim _{r \rightarrow \infty}(T r)=\text { const. or } \\
\lim _{r \rightarrow \infty} r(\text { attractional potential of Ell. })=G M \\
\lim _{r \rightarrow \infty}\left(T r^{2}\right)=0
\end{gathered}
$$

(Groten, 1979)

$$
\begin{gathered}
T / \gamma=\overline{S E}=N(\text { geoid height }) \\
-\vec{\gamma}=\nabla U .
\end{gathered}
$$

In other words, with respect to a level ellipsoid $E$, as defined above, we can determine the geoid where the volume of the ellipsoid is the same as the geoidal volume and the mass $M(E)$ of the level ellipsoid $E$ equals the mass of the Earth ( $M$, including the atmospere of the Earth; $M_{A}=$ mass of the atmosphere is $\left.10^{-6} M\right)$.

The practical determination of the geoid involves the solution of an oblique boundary value problem of elliptic type 


$$
\Delta T=0
$$

outside the Earth's surface $\left(S^{\prime}\right)$; the separation $\left(\overline{S^{\prime} S}\right)$ is called orthometric height.

$\Delta T=0$ is Laplace's equation, (6) defines a conservative force, (5) is Bruns' equation. The fixed type of (7) is based on boundary values $\delta g\left(S^{\prime}\right)=g\left(S^{\prime}\right)-$ $\gamma\left(S^{\prime}\right)$ where

$$
-\vec{g}=\nabla W
$$

thus $W^{o}$ is the key quantity which may be connected to $U_{g}$ (related to terrestrial time TT), $U_{g}$ being directly referred to $L_{g}$ in the definition of atomic time in relativistic theory.

Inside $S^{\prime}$ the disturbing potential fulfills Poisson's equation

$$
\Delta=-4 \pi G d
$$

where $d=$ density. Thus the determination of the geoid is equivalent to the determination of

$$
S=W^{o}(r, \theta, \lambda)
$$

and usually linear perturbation theory can be applied. The alternative altimetrygravimetry problem was outlined in (Groten, 1976).

One fundamental problem with such a solution is due to the fact that in $U$ the parameters $G M, a$ and $W^{o}$ are no longer derived as independent quantities and $J_{2}$ depends significantly on the underlying specific tidal regime. Equations (1) to (7) basically describe the definition, the remaining part basically the implementation of $S$.

\section{References}

Andersen, O.B. 2000, The geodesist's handbook, J. Geodesy, 74, 1

Groten, E. 1976, in Betr. Grav. Geodäsie und Gezeitenforschung, E. Groten, ed., DGK B 217, 99

Groten, E. 1979, Geodesy and the Earth's gravity field, vol. I, Dümmler-Verlag, Bonn

Groten, E. 2000, Z. Vermessungsw., 1, 1

Mathews, P.M., Herring, T.A., Buffett, B.A. 2000, Modelling of nutation-precession: New nutation series for non-rigid earth, and insights into the Earth's interior, presented at IAU Coll. 180 